\title{
Nitidine chloride inhibits the malignant behavior of human glioblastoma cells by targeting the PI3K/AKT/mTOR signaling pathway
}

\author{
MING LIU ${ }^{1}$, JIWEI WANG ${ }^{1}$, QICHAO QI ${ }^{1}$, BIN HUANG $^{1}$, ANJING CHEN $^{1}$, XINGANG LI ${ }^{1}$ and JIAN WANG ${ }^{1,2}$ \\ ${ }^{1}$ Department of Neurosurgery, Qilu Hospital of Shandong University and Brain Science Research Institute, \\ Shandong University, Wenhua Xi Road, Jinan, Shandong 250012, P.R. China; \\ ${ }^{2}$ Department of Biomedicine, University of Bergen, 5009 Bergen, Norway
}

Received May 16, 2016; Accepted June 13, 2016

DOI: $10.3892 /$ or.2016.4998

\begin{abstract}
Recent studies have demonstrated that nitidine chloride (NC), a natural bioactive alkaloid, displays potent antitumor activity in various types of cancer. In the present study, $\mathrm{NC}$ was examined for efficacy in the treatment of human glioblastoma multiforme (GBM) as well as the molecular basis for its more general inhibitory effects in cancer. U251 and U87 GBM cell lines were exposed to three concentrations of $\mathrm{NC}(5,25$ and $50 \mu \mathrm{M})$ in vitro, and tumor cell growth was assessed on the basis of proliferation, migration and energy metabolism. Decreases in viability and proliferation reached $\sim 50 \%$ for both cell lines with $50 \mu \mathrm{M} \mathrm{NC}$ at $24 \mathrm{~h}$ as assessed by cell viability Cell Counting Kit-8 (CCK-8) and EdU assays. In wound closure and Transwell assays, migration and invasion were inhibited at $50 \mu \mathrm{M}$ after $24 \mathrm{~h}(\sim 20$ and $80 \%$, respectively; $\mathrm{P}<0.05)$. ATP and L-lactate levels were also decreased after treatment with $\mathrm{NC}(50 \mu \mathrm{M}, 24 \mathrm{~h} ; \mathrm{P}<0.05$ and $\mathrm{P}<0.01$, respectively). Finally, in western blot analysis, phosphorylation of Akt and mTOR was suppressed by NC, but partially restored when cells were treated simultaneously with a novel Akt activator, SC79. Partial restoration was also observed in viability/ proliferation (U251 and U87, 15 vs. 40\%; NC vs. NC + SC79; $\mathrm{P}<0.05$ ) and invasion (U251 and U87, 30 vs. 60\%; NC vs. $\mathrm{NC}+\mathrm{SC79} ; \mathrm{P}<0.05)$. Our results demonstrated that $\mathrm{NC}$ inhibits development of GBM by targeting the PI3K/Akt/ mTOR signaling pathway and provides a potential therapeutic agent for the treatment of GBM.
\end{abstract}

Correspondence to: Dr Xingang Li or Dr Jian Wang, Department of Neurosurgery, Qilu Hospital of Shandong University and Brain Science Research Institute, Shandong University, Wenhua Xi Road, Jinan, Shandong 250012, P.R. China

E-mail:lixg@sdu.edu.cn

E-mail: jian.wang@uib.no

Key words: apoptosis, glioblastoma, metabolism, nitidine chloride, SC79

\section{Introduction}

Glioblastoma multiforme (GBM) is the most common malignant and aggressive primary human brain tumor (1). It is highly proliferative, invasive and chemoresistant, thus, that even with the combined treatment of surgery, chemotherapy and radiotherapy, the 5-year survival rate of WHO grade IV glioblastoma remains at less than $5 \%(2,3)$. Although significant advances have been made in our understanding of the molecular status of this tumor type, novel efficacious therapeutic avenues are critically needed.

Natural products have received recent interest in the discovery of novel anticancer therapeutic agents as have long been used as alternative remedies for a variety of diseases including cancer with relatively few side-effects $(4,5)$. Nitidine chloride (NC), a natural bioactive phytochemical alkaloid, has displayed anticancer activity in various types of cancer, such as ovarian, colorectal, hepatic and renal cancers (6-9). However, whether NC exhibits tumor inhibiting activity on GBM cells and the molecular mechanisms that underlie its tumoricidal activity remain largely unknown.

In the present study, GBM cell lines were exposed to varying concentrations of $\mathrm{NC}$ in vitro, and efficacy was evaluated in functional assays for proliferation, migration and invasion, and apoptosis. Alterations in metabolism and mitochondrial function were also investigated as well as changes in potential molecular targets in order to understand the basis of its activity. Our results revealed NC as a potential therapeutic agent in the treatment of human GBM and that the molecular basis for tumor inhibition may be through targeting of the $\mathrm{PI} 3 \mathrm{~K} / \mathrm{Akt} / \mathrm{mTOR}$ signaling pathway.

\section{Materials and methods}

Cell lines and cultures. Human glioma cell lines (U251 and U87) were purchased from the Chinese Academy of Sciences Cell Bank (Shanghai, China). Both U251 and U87 have been recently authenticated through cross species checks, DNA fingerprinting and quarantine. Cells were grown in Dulbecco's modified Eagle's medium (DMEM; SH30022.01B; Gibco, GE Healthcare Life Sciences, Pittsburgh, PA, USA) supplemented 
with $10 \%$ fetal bovine serum (FBS) (10082147; HyClone, GE Healthcare Life Sciences) in a humidified incubator with $5 \% \mathrm{CO}_{2}$ at $37^{\circ} \mathrm{C}$.

Cell viability and proliferation assays. Cell viability was assessed with the Cell Counting Kit-8 (CCK-8; CK04-500; Dojindo, Kumamoto, Japan). Tumor cells (1.0x $10^{4}$ cells/well) were seeded into 96-well, flat-bottomed plates with DMEM containing $10 \% \mathrm{FBS}$ and incubated at $37^{\circ} \mathrm{C}$ overnight. Nitidine chloride (NC) (N117977; Aladdin Biotech, Shanghai, China) or SC79 (SML0749) was dissolved in dimethyl sulfoxide (DMSO) (D2650) (both from Sigma-Aldrich, St. Louis, MO, USA) and diluted to working concentrations in culture medium. After the desired treatment, the cells were incubated for an additional $4 \mathrm{~h}$ at $37^{\circ} \mathrm{C}$ with $10 \mu \mathrm{l}$ of CCK-8 in $100 \mu \mathrm{l}$ of serum-free DMEM. The absorbance at $450 \mathrm{~nm}$ was measured using a microplate reader (Bio-Rad, Hercules, CA, USA). Proliferation was assessed using the EdU incorporation assay according to the manufacturer's protocol (C103103; RiboBio, Guangzhou, China). Briefly, EdU was incorporated into the proliferating cells and was detected through a catalyzed reaction with a fluorescently labeled azide. The percentage of labeled cells (ratio: $\mathrm{EdU}^{+} / \mathrm{DAPI}^{+}$x $100 \%$ ) was determined using images captured in four random fields per sample under fluorescence.

Cell migration and invasion assays. Cell migration was assessed in wound healing assays. U251 or U87 glioma cells $\left(1 \times 10^{5} /\right.$ well $)$ were seeded into 6 -well plates and incubated overnight. A cell-free gap was generated by scratching the cell monolayer with a $200 \mu \mathrm{l}$ pipette tip. The scratched plates were cultured in DMEM containing 1\% FBS, and images used for analysis were captured under a light microscope at 0 and $24 \mathrm{~h}$. Cell invasion was examined using the Transwell chamber assay. The bottom of the Transwell membrane was pretreated with Matrigel (Becton-Dickinson, Bedford, MA, USA) for $4 \mathrm{~h}$. Cells $\left(5 \times 10^{4}\right)$ were re-suspended in serum-free DMEM and seeded into the upper chamber of a Transwell apparatus (8.0- $\mu \mathrm{m}$ pore; Corning, Sigma-Aldrich). DMEM containing $10 \%$ FBS $(600 \mu \mathrm{l})$ was added to the lower chamber, and chambers were incubated at $37^{\circ} \mathrm{C}$ for $24 \mathrm{~h}$. Cells that had migrated to the bottom of the membrane after $24 \mathrm{~h}$ were fixed and stained with crystal violet for $15 \mathrm{~min}$, while those remaining in the upper chamber were removed with a cotton swab. Five random views from images acquired under a light microscope were used to count the migrated cells.

\section{Measurement of mitochondrial membrane potential $(\Delta \psi m)$} and mitochondrial morphology. Live cells were seeded into 96-well plates and loaded with tetramethylrhodamine methyl ester (JC-1) for 30 min according to the manufacturer's protocol. Fluorescence was measured in a microplate reader, and the fluorescence intensity ratio of JC-1 aggregates to JC-1 monomers [ratio of $590 \mathrm{~nm}: 530 \mathrm{~nm}$ emission intensities; (10)] was used to determine the $\Delta \psi \mathrm{m}$. For mitochondrial morphology, live cells were seeded in 6-well plates and fluorescently labeled with $25 \mathrm{nM}$ MitoTracker Red (Molecular Probes, Thermo Fisher Scientific, Waltham, MA, USA). In both analyses, cells were examined under an Olympus BX61 fluorescence microscope, and images were acquired using a DP71 charge-coupled device (CCD) digital camera (Olympus, Waltham, MA, USA).

Western blotting. U251 and U87 glioma cells were harvested, rinsed with phosphate-buffered saline (PBS) and lysed with RIPA buffer (P0013B; Beyotime, Shanghai, China) containing $1 \%$ phenylmethylsulfonyl fluoride. Protein concentrations were determined using the BCA method (23225; Beyotime). Proteins $(20 \mu \mathrm{g})$ were separated on 8-15\% SDS-PAGE and transferred onto polyvinylidene fluoride (PVDF) membranes (ISEQ00010 $0.22 \mu \mathrm{m}$; Millipore, Billerica, MA USA). Membranes were blocked for $2 \mathrm{~h}$ at room temperature (RT) with 5\% non-fat dry milk in TBST [ $20 \mathrm{mmol} / \mathrm{l}$ Tris-HCL (pH 8.0), $137 \mathrm{mmol} / 1 \mathrm{NaCl}$ and $0.1 \%$ Tween-20 or with $5 \%$ BSA in TBST for phospho-proteins], incubated with primary antibodies overnight at $4^{\circ} \mathrm{C}$, rinsed with TBST, and probed with horseradish peroxidase (HRP)-conjugated secondary antibody (1:5,000; Santa Cruz Biotechnology, Dallas, TX, USA) for $1 \mathrm{~h}$ at RT. Proteins were visualized with enhanced chemiluminescence (ECL; Millipore) and the ChemiDoc Touch detection system (Bio-Rad). The following primary antibodies were used: rabbit anti-cytochrome $c, \mathrm{Bcl}-2, \mathrm{Drp}-1, \mathrm{AKT}, \mathrm{p}-\mathrm{Akt}$ (Ser473), mTOR, p-mTOR (Ser2448), GAPDH (Cell Signaling Technology, Danvers, MA, USA), rabbit anti-mitofusion-1 and Bax (Abcam, Cambridge, MA, USA).

Measurement of ATP and lactate. Cells (2x105/well) were seeded into 6-well plates. After incubation overnight, the culture medium supernatants were collected for the determination of the concentration of lactate. Cells were subsequently trypsinized and counted for ATP concentrations and normalization. Total ATP and lactate concentrations were determined using the CellTiter-Glo Luminescent assay (Promega, Madison, WI, USA) and the L-lactate colorimetric assay kit (Abcam), respectively, according to the manufacturer's protocols. Data were normalized to the number of cells.

Immunofluorescence. U251 and U87 cells were fixed with 4\% formaldehyde in PBS, permeabilized with $0.5 \%$ Triton X-100 in PBS, incubated with rabbit anti-cytochrome $c$ antibody (1:400) in 5\% bovine serum albumin in PBS overnight, and labeled with FITC- and DyLight 594-conjugated anti-rabbit IgG (Santa Cruz Biotechnology). Cells were incubated in the dark with Rhodamine phalloidin (PHDR1; Cytoskeleton, Inc., Denver, CO, USA) and DAPI to stain actin and nuclei, respectively. Slides were examined under an Olympus BX61 fluorescence microscope, and images were acquired using a DP71 CCD digital camera.

Flow cytometric analysis of apoptosis. U251 and U87 glioma cells were harvested, re-suspended in binding buffer, and incubated with Annexin V-FITC (BD Biosciences, Franklin Lakes, NJ, USA) according to the manufacturer's instructions. Apoptotic cells were detected by flow cytometry (NovoCyte Flow Cytometer; ACEA Biosciences, San Diego, CA, USA), and the results were analyzed using the software FlowJo (Tree Star, Ashland, OR, USA).

Statistical analysis. Three independent experiments were performed and the results are expressed as the mean \pm standard 


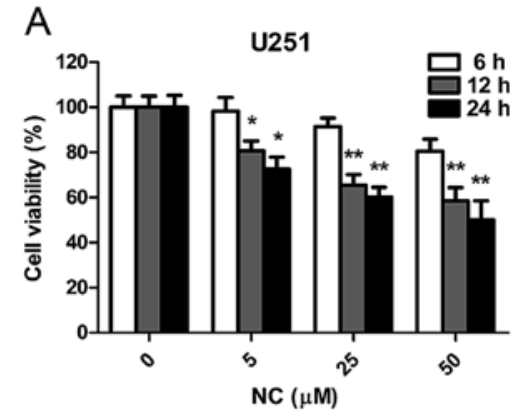

B
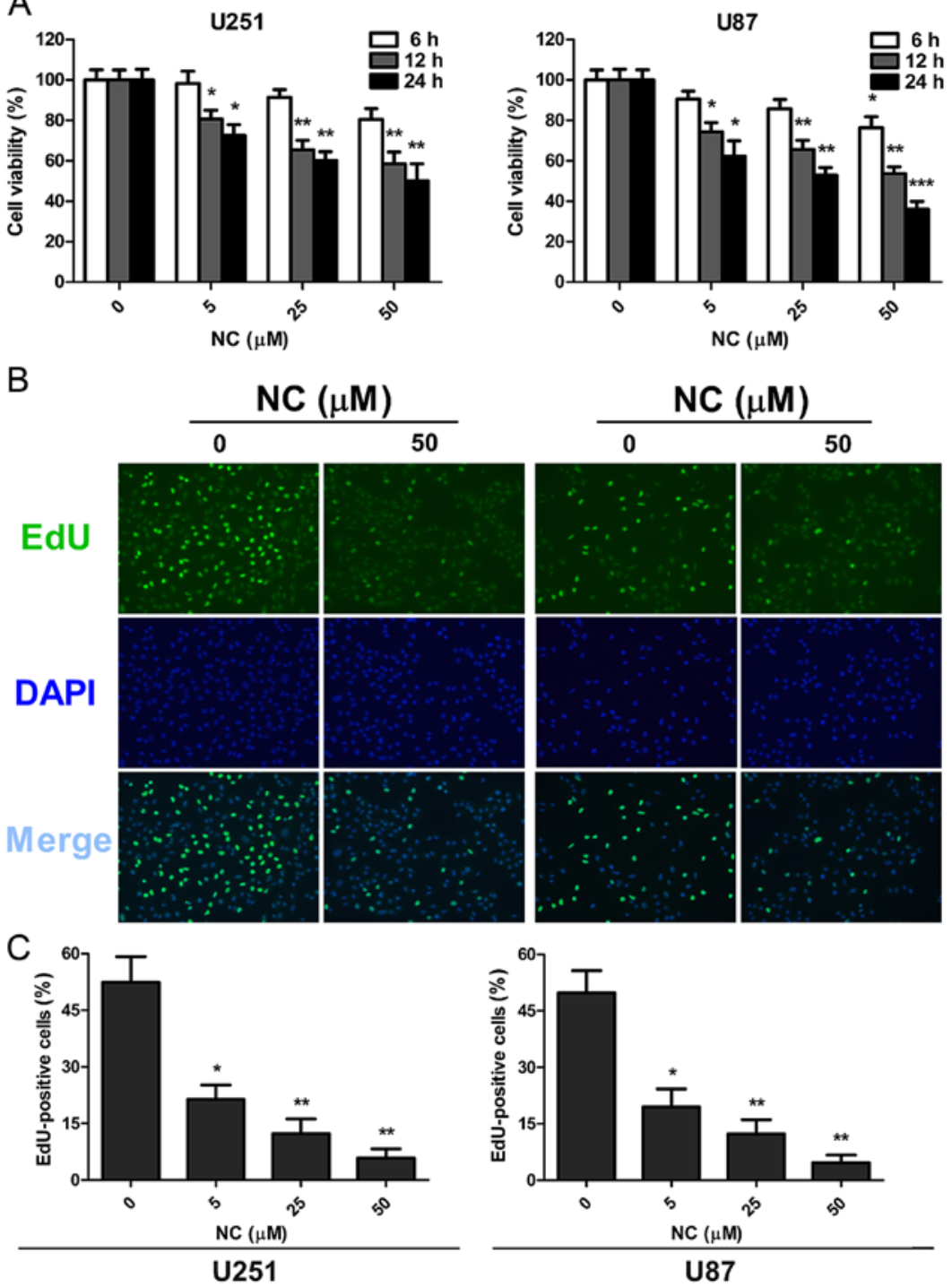

Figure 1. Nitidine chloride (NC) inhibits the growth of GBM cells. (A) Viability as determined in CCK-8 assays performed on U251 and U87 cells treated with different concentrations of $\mathrm{NC}$ for 6,12 and $24 \mathrm{~h}$. Results are presented as a percentage (\%) relative to the untreated cells at that time point. (B) Fluorescent images of EdU incorporation in U251 and U87 cells treated with NC or DMSO for 24 h. Cells were stained with Apollo 488 (green) to detect EdU and DAPI (blue) to highlight nuclei and images were superimposed. (C) Cell number and EdU content of U251 and U87 cells treated with different concentrations of $\mathrm{NC}$ for $24 \mathrm{~h}$. The percentage of EdU ${ }^{+}$cells $\left(\mathrm{EdU}^{+} / \mathrm{DAPI}^{+} \mathrm{x} 100 \%\right)$ was determined in four random fields per sample. All data are expressed as the mean $\pm \mathrm{SD}$ of values from experiments performed in triplicate; ${ }^{*} \mathrm{P}<0.05,{ }^{* *} \mathrm{P}<0.01$ and ${ }^{* * *} \mathrm{P}<0.001$ compared to the controls.

deviation (SD). Data were compared using paired t-tests in GraphPad Prism 5 software (San Diego, CA, USA), and $\mathrm{P}$-values determined from different comparisons are indicated in the figures as follows: ${ }^{*} \mathrm{P}<0.05,{ }^{* *} \mathrm{P}<0.01$ and ${ }^{* * *} \mathrm{P}<0.001$.

\section{Results}

NC inhibits proliferation of GBM cells. To determine whether $\mathrm{NC}$ may be effective against GBM, NC treatment was first evaluated in $\mathrm{U} 87$ and $\mathrm{U} 251$ cells in vitro, using the cell viability assay, CCK-8 (Fig. 1A). Cells were treated with differing concentrations of $\mathrm{NC}$ in vitro, and viability was assessed at 6 , 12 and $24 \mathrm{~h}$. Decreases in cell viability relative to the untreated cells were statistically significant at the 12 and $24 \mathrm{~h}$ time points at all concentrations of NC. The most dramatic decrease in viability was $\sim 50 \%$ for both cell lines following treatment with $50 \mu \mathrm{M}$ NC at $24 \mathrm{~h}$.
EdU incorporation was used to further evaluate proliferation of the GBM cell lines in the presence of NC. Quantification of EdU incorporation revealed a statistically significant decrease in proliferation for both cell lines at $24 \mathrm{~h}$ after exposure to $\mathrm{NC}$ at all concentrations. The NC concentration of $50 \mu \mathrm{M}$ however was the most effective in both cell lines ( 50 vs. $5 \%$; untreated vs. treated cells). These results indicated that NC potently arrested proliferation in both the U251 and U87 cells and in a dose-dependent manner (Fig. 1B and C).

$N C$ attenuates migration and invasion of GBM cells. To determine whether NC inhibits the migration and invasive properties of GBM cells, wound healing and invasion assays were performed on U251 and U87 cells in the presence of different concentrations of NC. Wound closure at $24 \mathrm{~h}$ in monolayer culture was inhibited at all NC concentrations for both U251 and U87 cells (Fig. 2A and B). Increasing NC concentrations 
A

$\mathrm{NC}(\mu \mathrm{M})$

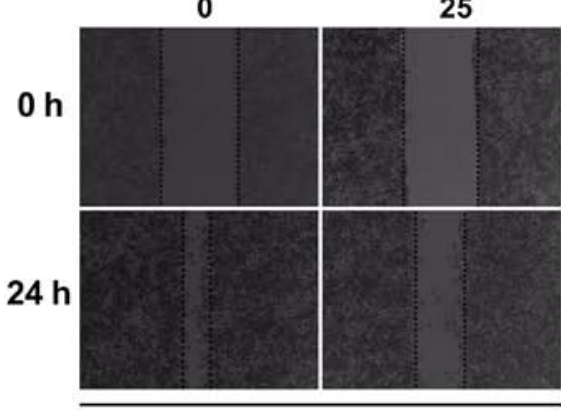

U251

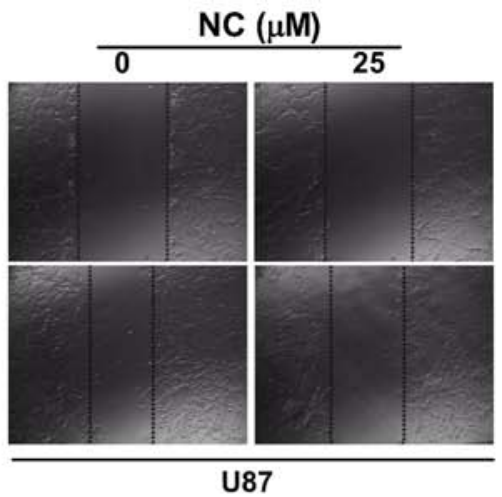

B
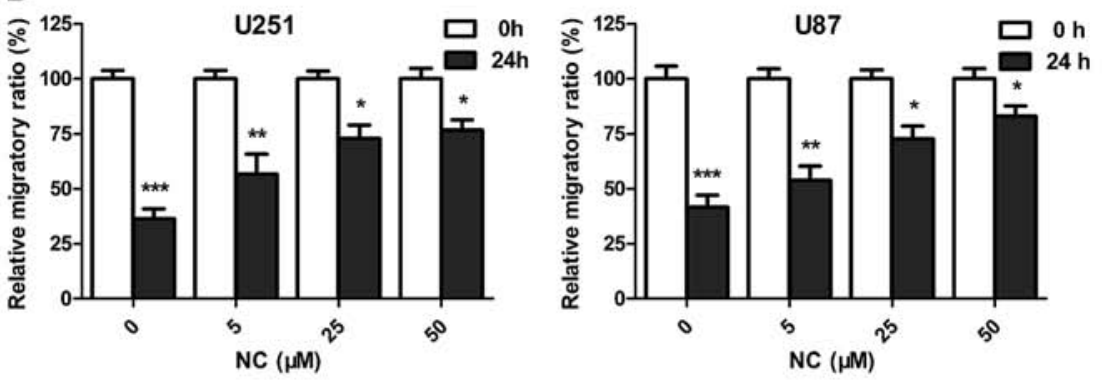

C

$\mathrm{NC}(\mu \mathrm{M})$
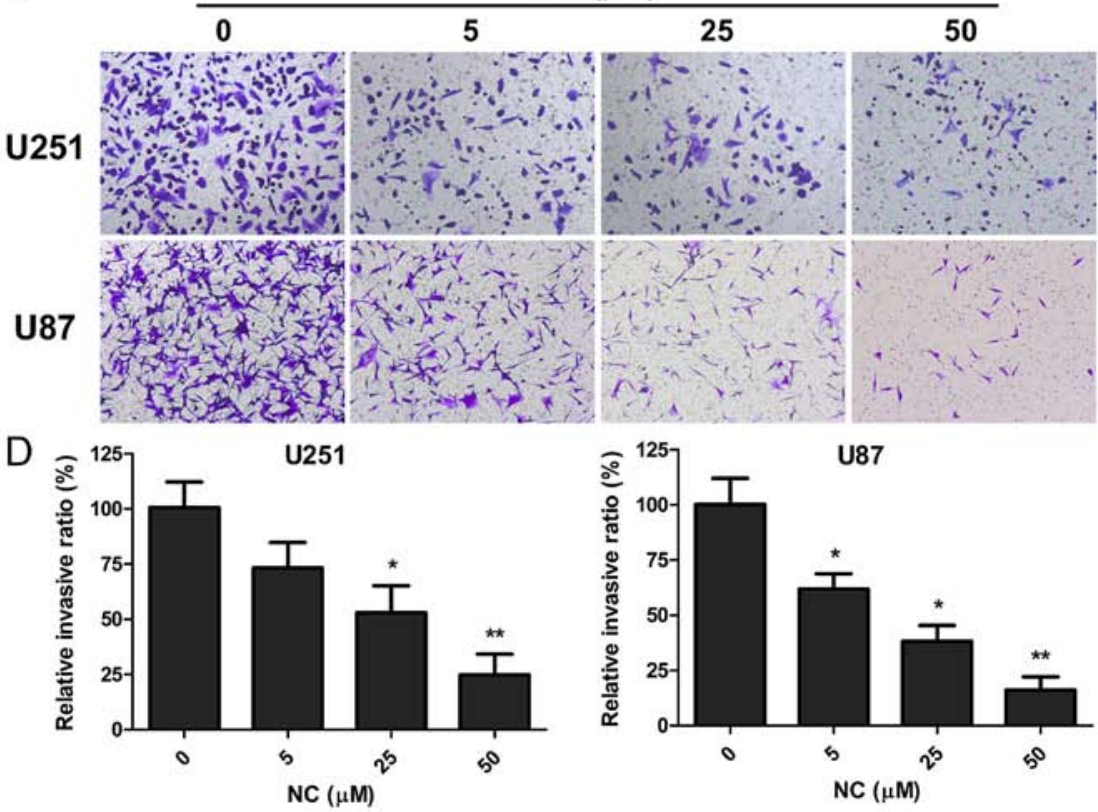

Figure 2. Nitidine chloride (NC) attenuates migration and invasion in GBM cells. (A) Images of wound closure assays (24 h) for U251 and U87 cells treated with $25 \mu \mathrm{M}$ NC or DMSO for $24 \mathrm{~h}$. (B) Statistical results for the wound closure area treated with different concentrations of NC for $24 \mathrm{~h}$. (C) Transwell membranes for U251 and U87 cells treated with different concentrations of NC for $24 \mathrm{~h}$ fixed and stained with crystal violet. (D) Statistical results of the invasive ratio for U251 and U87 cells treated with different concentrations of NC for $24 \mathrm{~h}$ in the Transwell assay. All data are expressed as the mean \pm SD of values from experiments performed in triplicate; ${ }^{*} \mathrm{P}<0.05,{ }^{* *} \mathrm{P}<0.01$ and ${ }^{* * *} \mathrm{P}<0.001$ compared to the controls.

were more effective, with only partial wound closure at $50 \mu \mathrm{M}$ after $24 \mathrm{~h}(\sim 20 \%$; $\mathrm{P}<0.05)$. The Transwell assay was used to further evaluate the effect of $\mathrm{NC}$ treatment on the invasion of GBM cells. Upper chambers of Transwell apparatuses were coated with Matrigel in order to provide extracellular matrix (ECM) simulating a normal tumor microenvironment, and seeded with U251 or U87 cells. Counts of cells that had migrated through the membrane at $24 \mathrm{~h}$ were decreased with increasing concentrations of NC. At $50 \mu \mathrm{M}$, cell counts were decreased by $\sim 80 \%$ for both cell lines (Fig. 2C and D). These results indicate that $\mathrm{NC}$ profoundly inhibited migration and invasion of $\mathrm{U} 251$ and $\mathrm{U} 87$ cells in vitro.

NC inhibits production of ATP and L-lactate in GBM cells. Tumor cell invasion is a high energy and nutrient consuming process $(11,12)$, with increased glycolysis as one of the most prominent metabolic alterations universally occurring in cancer cells (13). Molecules that may uncouple glycolysis have thus been the basis for several treatment strategies for cancer. To begin to understand whether NC may interfere with cancer 


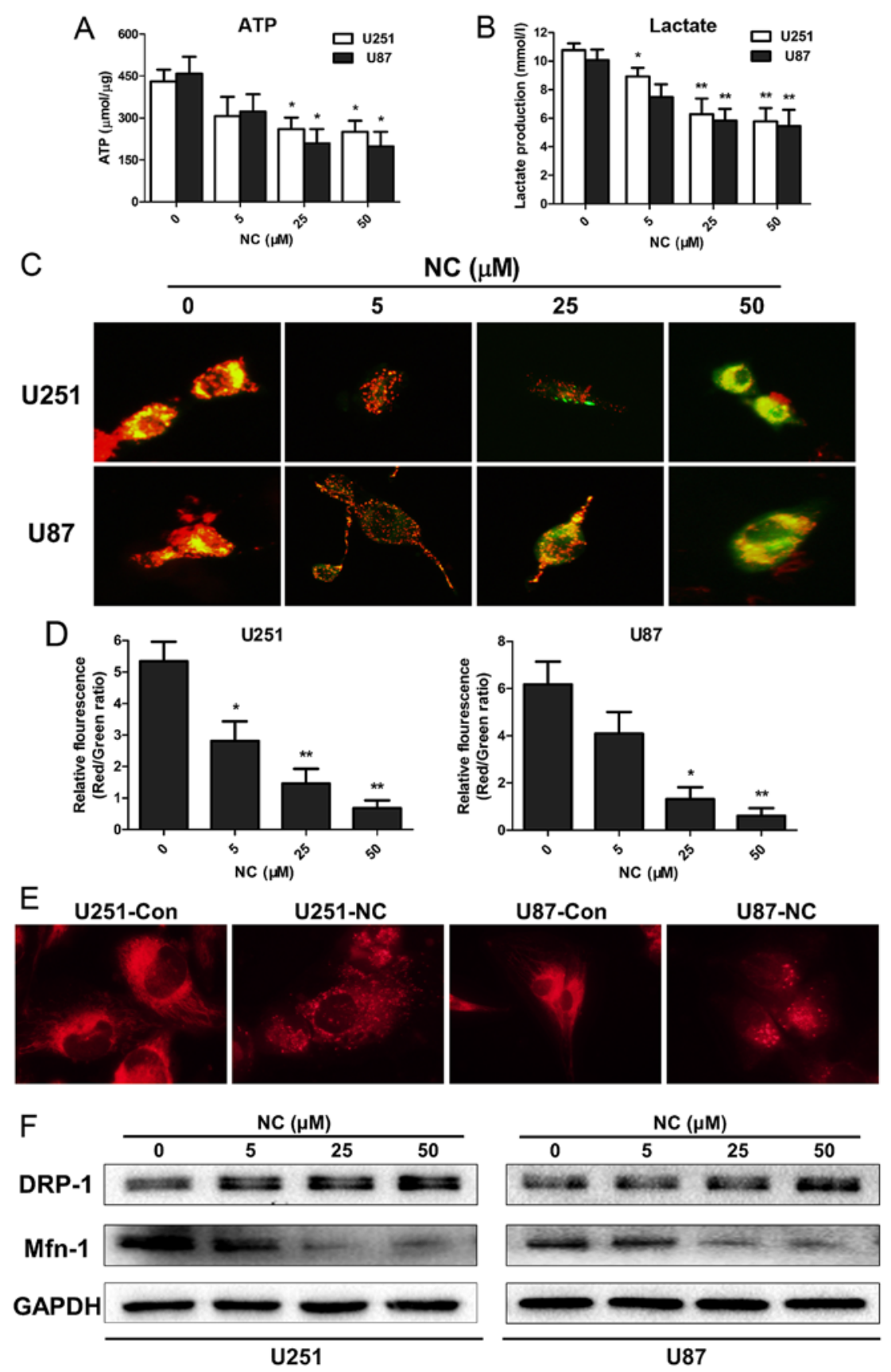

Figure 3. Nitidine chloride (NC) inhibits glycolysis and disrupts mitochondrial function in GBM cells. (A) ATP synthesis levels in U251 and U87 cells treated with different concentrations of $\mathrm{NC}$ for $24 \mathrm{~h}$. (B) L-lactate concentrations in media collected from U251 and U87 cells treated with different concentrations of $\mathrm{NC}$ for $24 \mathrm{~h}$. (C) Representative images of U251 and U87 cells incubated with the JC-1 probe to determine $\Delta \psi \mathrm{m}$ following treatment with different concentrations of NC for $24 \mathrm{~h}$. Images were acquired under different wavelengths and superimposed. (D) $\Delta \psi \mathrm{m}$ quantitated by flow cytometry as a function of the red/green ratio of JC-1 probe as aggregates or monomers. (E) MitoTracker Red staining for mitochondria in U251 and U87 cells treated with NC (50 $\mu \mathrm{M})$ or DMSO for $24 \mathrm{~h}$. (F) Western blot analysis of Mfn-1, Drp-1 and GAPDH (protein loading control) in the U251 and U87 cells treated with different concentrations of $\mathrm{NC}$ for $24 \mathrm{~h}$. All data are expressed as the mean $\pm \mathrm{SD}$ of values from experiments performed in triplicate; ${ }^{*} \mathrm{P}<0.05,{ }^{* * *} \mathrm{P}<0.01$ and ${ }^{* * *} \mathrm{P}<0.001 \mathrm{compared}$ to the controls.

cell metabolism, ATP synthesis levels were first examined in the NC-treated GBM cells. In both the U251 and U87 cells, ATP levels were moderately decreased after treatment with $\mathrm{NC}$ at 25 and $50 \mu \mathrm{M}$ concentrations at $24 \mathrm{~h}(\mathrm{P}<0.05$; Fig. $3 \mathrm{~A})$. L-lactate is the main metabolic product generated in glycolysis, and high levels are typically observed in cell culture media collected from cancer cells. L-lactate concentrations in cell media from U251 and U87 cells were therefore also evaluated under treatment with $\mathrm{NC}$ at $24 \mathrm{~h}$. Maximum decreases in
L-lactate concentrations were found in media from both U251 and U87 cells following NC treatment at $25 \mu \mathrm{M}$ with no further enhancement at $50 \mu \mathrm{M}(\mathrm{P}<0.01)$. These results indicated that $\mathrm{NC}$ treatment suppressed glycolysis in the GBM cells (Fig. 3B).

Mitochondrial function is altered in GBM cells treated with $N C$. Mitochondria are the key organelles generating ATP in the cell, and the $\Delta \psi \mathrm{m}$ is a critical factor in the production of 

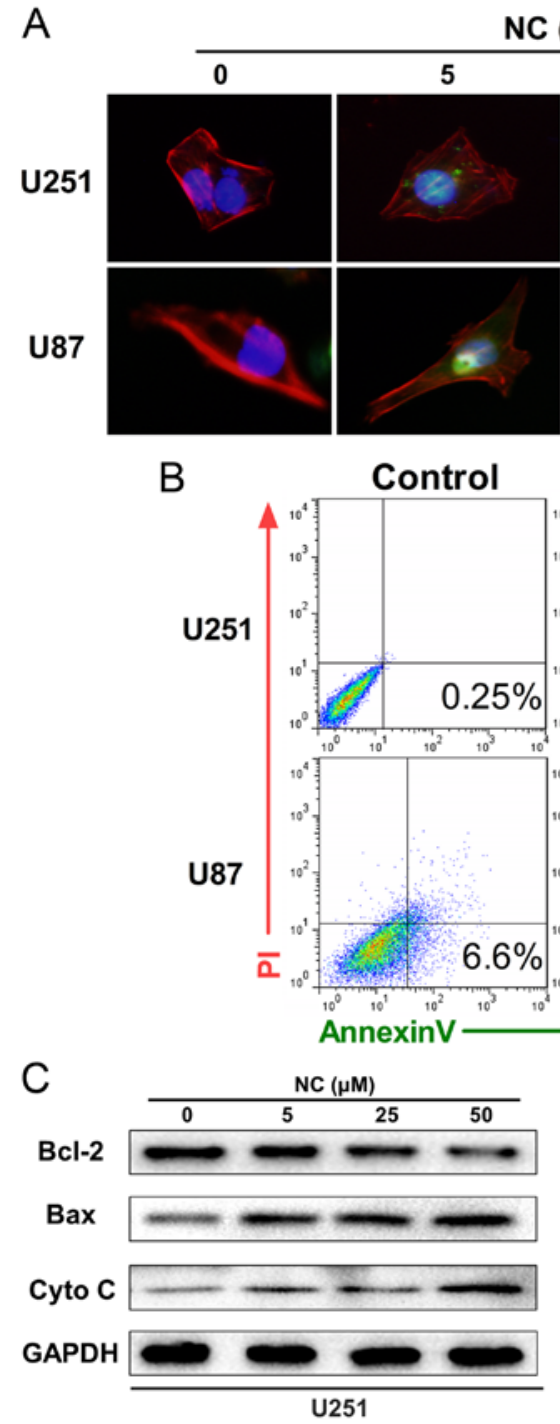

NC $(\mu \mathrm{M})$
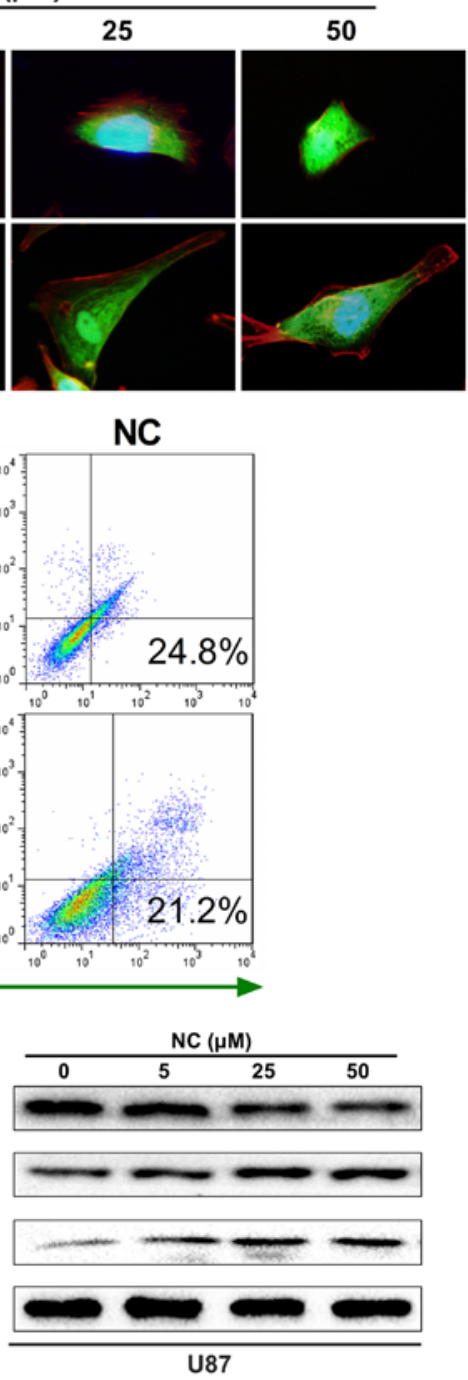

Figure 4. Nitidine chloride (NC) induces mitochondrial apoptosis in GBM cells. (A) Immunofluorescent imaging (IFI) to localize cytochrome $c$ in the U251 and U87 cells treated with different concentrations of NC for $24 \mathrm{~h}$. (B) Annexin V/PI staining of U251 and U87 cells treated with NC (50 $\mu$ M) for 24 h as analyzed by flow cytometry. (C) Western blot analyses for Bax, Bcl-2, cytochrome(Cyto) $c$ and GAPDH in the U251 and U87 cells treated with different concentrations of $\mathrm{NC}$ for $24 \mathrm{~h}$. All data are expressed as the mean $\pm \mathrm{SD}$ of values from experiments performed in triplicate; ${ }^{*} \mathrm{P}<0.05,{ }^{* * *} \mathrm{P}<0.01$ and ${ }^{* * * *} \mathrm{P}<0.001$ compared to the controls.

ATP. Changes in $\Delta \psi \mathrm{m}$ detected on the basis of fluorescence from the JC-1 probe can be a signal of reduced mitochondrial function. The JC-1 probe is an indicator of $\Delta \psi \mathrm{m}$, which emits red fluorescence upon formation of J-aggregates under high $\Delta \psi \mathrm{m}$ conditions and green fluorescence from J-monomers under low $\Delta \psi \mathrm{m}$ conditions. Thus, the conversion between red and green fluorescence directly reflects changes in $\Delta \psi \mathrm{m}$ (14). In the U251 and U87 cells, green fluorescence was increased following treatment with $\mathrm{NC}$ at 25 and $50 \mu \mathrm{M}$ concentrations (Fig. 3C) as demonstrated by the decrease in the red/ green fluorescence ratios (Fig. 3D). These results indicate that mitochondrial activity was suppressed in the NC-treated U251 and U87 cells relative to the untreated cells.

ATP production has been associated with changes in mitochondrial morphology which largely occur due to a dynamic switch between fusion and fission states in mitochondria in order to accommodate diverse cellular scenarios (15). The fusion state, for example, has been found to be a more favorable form in cell invasion apices while the fission state was associated more often with cell trafficking (16). To determine whether mitochondrial morphology was altered under NC treatment, live cells were exposed to the fluorescent dye MitoTracker Red which is selective for the staining of mitochondria. The results demonstrated that the NC-treated cells favored the fission state, while in the untreated GBM cells the fusion state predominated (Fig. 3E).

Mitochondrial fission and fusion status is also accompanied by changes in proteins specifically associated with each of these processes. The protein Drp-1, for example, is essential for mitochondrial fission, while the protein Mfn-1 is required for mitochondrial fusion (17). To determine whether the levels of these proteins paralleled the morphological changes observed, western blot analyses were performed with lysates prepared from the U251 and U87 cells treated with NC at different concentrations for $24 \mathrm{~h}$. Protein levels of Drp-1 remained unchanged at all concentrations of NC. Mfn-1 protein levels were however decreased by $\sim 2$-fold at 25 and $50 \mu \mathrm{M} \mathrm{NC}$ concentrations (Fig. 3F). Taken together, these results indicate 

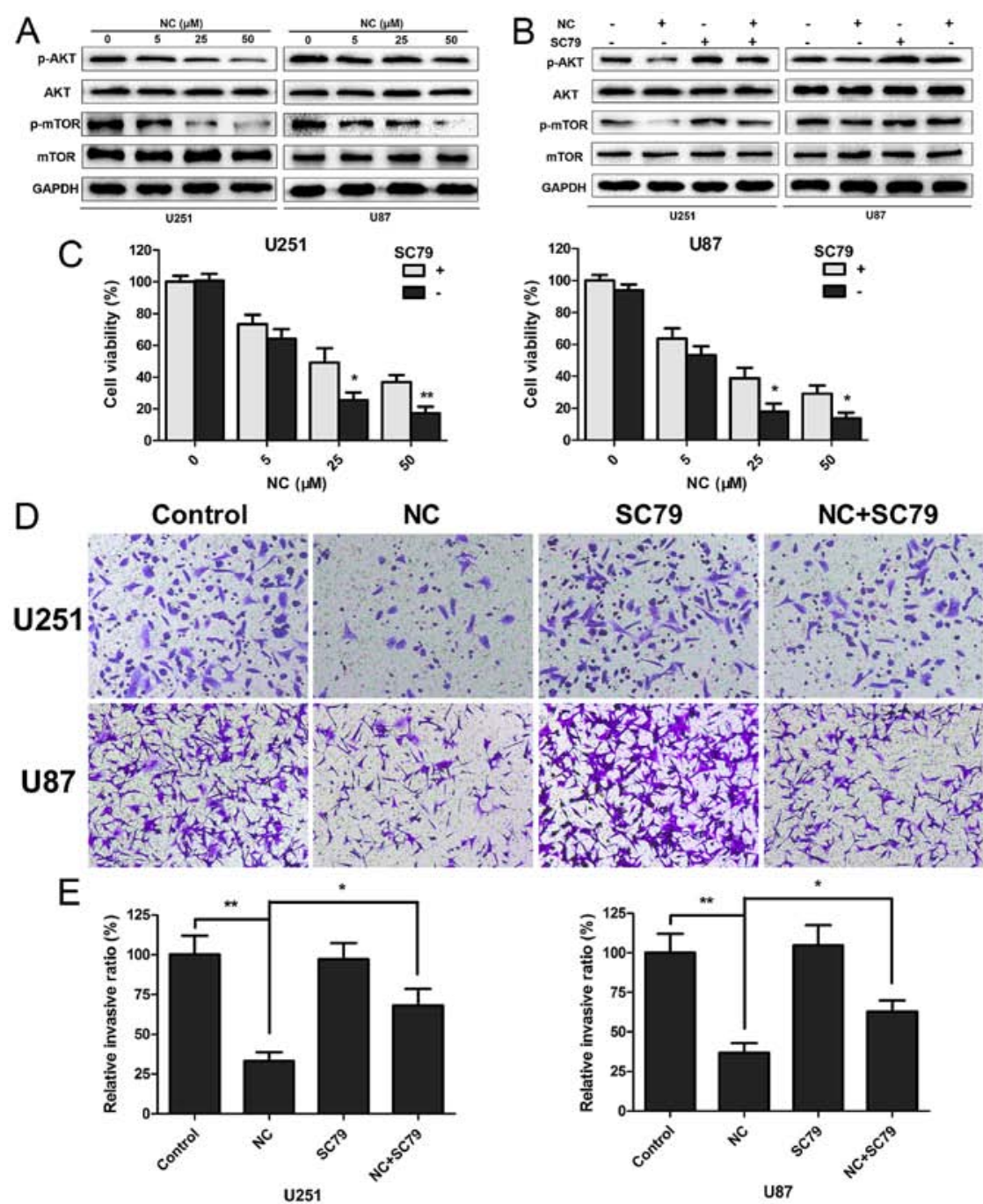

SC79

$\mathrm{NC}+\mathrm{SC} 79$
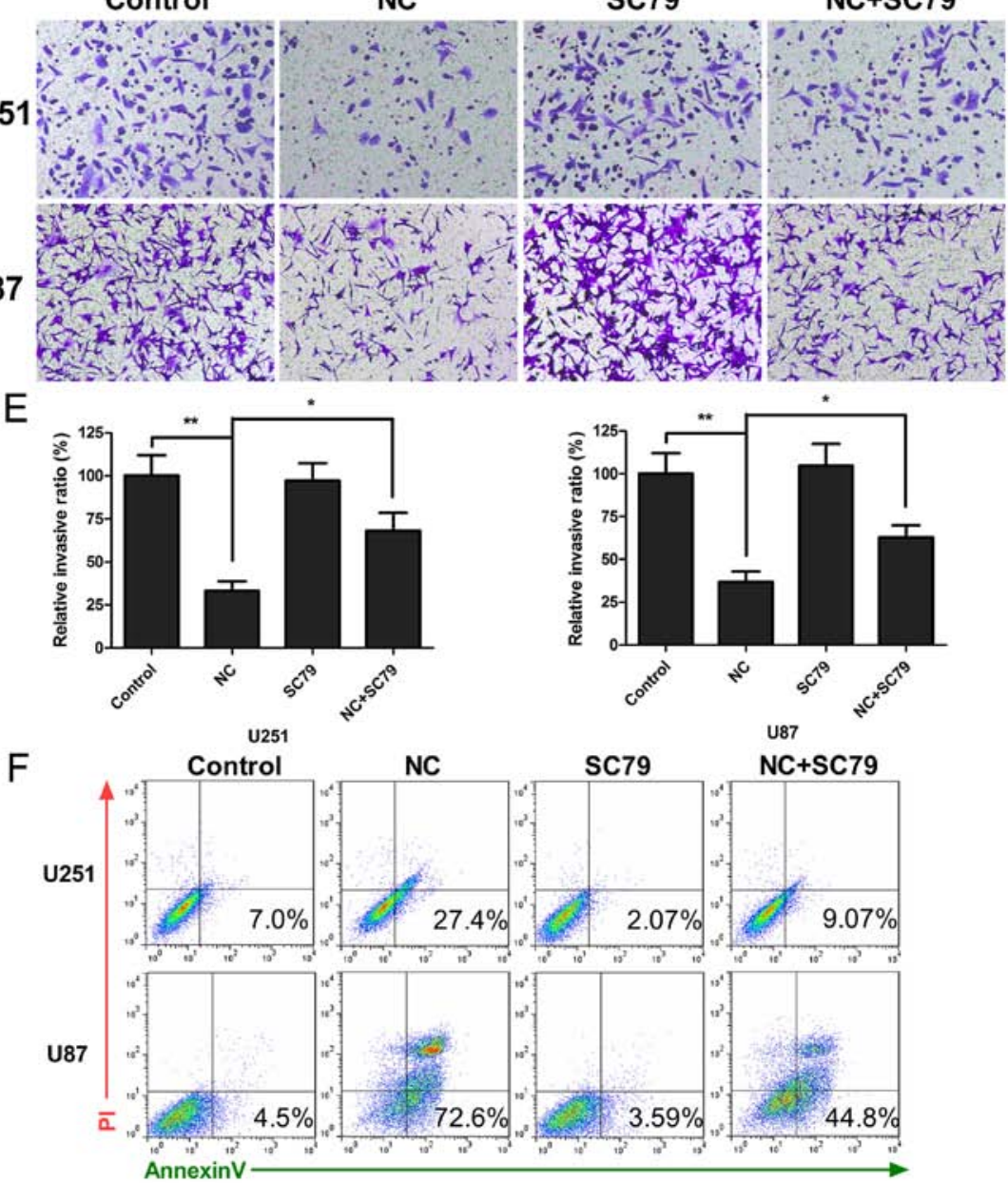

Figure 5. Nitidine chloride (NC) targets the PI3K/Akt/mTOR signaling pathway in GBM cells. (A) Western blot analysis of p-Akt, Akt, p-mTOR, mTOR and GAPDH expression in the U251 and U87 cells treated with NC at the concentration indicated or DMSO for $24 \mathrm{~h}$. (B) Western blot analysis of p-Akt, Akt, p-mTOR, mTOR and GAPDH expression in the U251 and U87 cells treated with NC at $50 \mu \mathrm{M}$ or DMSO in the absence or presence of SC79 $(5 \mu \mathrm{g} / \mathrm{ml})$ for $24 \mathrm{~h}$. (C) CCK-8 assay to assess cell viability in the U251 and U87 cells treated with NC at $50 \mu \mathrm{M}$ or DMSO in the presence or absence of SC79 for $24 \mathrm{~h}$. (D) Transwell membranes fixed and stained with crystal violet. U251 and U87 cells were treated with NC at $50 \mu \mathrm{M}$ or DMSO in the presence or absence of SC79 for $24 \mathrm{~h}$. (E) Statistical results of the invasive ratio of the U251 and U87 cells treated with NC (50 $\mu \mathrm{M})$ or DMSO in the presence or absence of SC79 for $24 \mathrm{~h}$ in the Transwell assay. (F) Annexin V/PI staining of U251 and U87 cells treated with NC (50 $\mu \mathrm{M})$ or DMSO in the absence or presence of SC79 for $24 \mathrm{~h}$ as analyzed by flow cytometry. ${ }^{*} \mathrm{P}<0.05,{ }^{* * *} \mathrm{P}<0.01$ and ${ }^{* * *} \mathrm{P}<0.001$ compared to the controls.

that $\mathrm{NC}$ treatment resulted in the inhibition of glycolysis and a decrease in mitochondrial function through changes in $\Delta \psi \mathrm{m}$ and mitochondrial morphology in the GBM cells.

NC induces mitochondrial apoptosis in GBM cells. Studies have shown that mitochondrial fission renders cells more sensitive to apoptosis (17). Our results above revealed that the morphology of mitochondria transitioned from fusion to fission after exposure to NC. We therefore investigated whether NC induces mitochondrial apoptosis in GBM cells. Immunofluorescent imaging (IFI) was first performed in order to localize cytochrome $c$ which is released from mitochondria into the cytosol during apoptosis. Cytochrome $c$ was found to be increased in the cytosol in the NC-treated U251 and 
U87 cells, particularly at 25 and $50 \mu \mathrm{M}$ concentrations after $24 \mathrm{~h}$ (Fig. 4A). The percentage of cells undergoing apoptosis was subsequently determined through FACS analysis of Annexin V/PI staining. Increases in apoptosis were observed in both the U251 and U87 cells in early (Annexin $\mathrm{V}^{+} / \mathrm{PI}^{-}$) and late (Annexin $\mathrm{V}^{+} / \mathrm{PI}^{+}$) stages with $50 \mu \mathrm{M} \mathrm{NC}$ at $24 \mathrm{~h}$ (Fig. 4B).

Finally, western blot analysis was used to determine the expression levels of mitochondrial proteins executing apoptosis, including Bax and cytochrome $c$, as well as Bcl-2, which inhibits apoptosis, in the NC-treated cells. Treatment with NC led to increases in Bax and cytochrome $c$ and simultaneous decreases in Bcl-2 compared to the untreated U251 and U87 cells (Fig. 4C).

NC inhibits PI3K/Akt/mTOR signaling. Aberrant activation of the PI3K/Akt/mTOR signaling pathway is known to promote tumorigenesis, and mutations in the pathway frequently occur in human GBM. The phosphorylation status of Akt and mTOR proteins in the presence of $\mathrm{NC}$ was therefore examined by western blotting to determine whether the observed responses could be due to decreased PI3K/Akt/ mTOR signaling. Increasing concentrations of NC suppressed phosphorylation of both Akt and mTOR, indicating that PI3K/Akt/mTOR signaling was inhibited by NC (Fig. 5A). Activation of the pathway was partially restored when cells were treated simultaneously with a novel Akt activator, SC79 (18). SC79 increased phosphorylation of Akt and mTOR in the NC-treated cells, and thus further established PI3K/Akt/ mTOR signaling as a molecular target for NC suppression of cell growth (Fig. 5B). Functional assays, including CCK-8, Transwell migration/invasion and Annexin V/PI staining, were repeated in order to determine whether SC79 may restore cell growth and migration of GBM cells under NC treatment. SC79 led to increased viability/proliferation (U251 and U87, $\sim 15$ vs. $40 \%$; $\mathrm{NC}$ vs. $\mathrm{NC}+\mathrm{SC} 79 ; \mathrm{P}<0.05)$ and invasion (U251 and U87, 30 vs. 60\%; $\mathrm{NC}$ vs. $\mathrm{NC}+\mathrm{SC79}$; $\mathrm{P}<0.05)$ in the NC-treated GBM cells (Fig. 5C-E). In contrast, apoptosis was decreased (U251, 27.4 vs. 9.04\%; U87, 72.6 vs. $44.8 \%$, NC vs. $\mathrm{NC}+\mathrm{SC79}$; Fig. 5F). Taken together, our data indicated that changes in proliferation, invasion and apoptosis induced by NC were in part due to inhibition of PI3K/Akt/mTOR signaling in the GBM cells.

\section{Discussion}

Therapeutic compounds are desperately needed to improve the dismal survival outcome for glioblastoma multiforme (GBM) patients. Multiple studies have provided compelling evidence that nitidine chloride (NC) plays an anticancer role in various aggressive malignancies (7-9,19-21). In the present study, we demonstrated that NC has anticancer properties affecting many critical cellular processes by targeting the PI3K/Akt/ mTOR pathway in GBM cells.

Our focus was to identify new potential therapies for GBM. $\mathrm{NC}$ however has also been reported to inhibit proliferation of colorectal and hepatic cancer cells $(7,9)$, and to attenuate migration and invasion of breast and ovarian cancer cells $(8,22)$. We therefore chose to examine the functional and molecular pathways that are commonly altered among diverse cancers in order to understand the mechanistic basis of NC inhibition.
Metabolism has emerged as a potential therapeutic target due to the fact that increased glycolysis is one of the most prominent metabolic alterations common among cancer types (13). Lactate, the main product of glycolysis, also promotes metabolism in cancer cells (23). NC attenuated both ATP and lactate generation. Furthermore, mitochondria, which are the key organelles for the generation of ATP, exhibited changes upon exposure to $\mathrm{NC}$, including lower $\Delta \psi \mathrm{m}$ and unbalanced mitochondrial fusion and fission. Such decreases in mitochondrial output and function in response to NC may ultimately lead to the inhibition of tumor cell migration and invasion which are high-energy consuming processes.

Disruptions in mitochondrial fusion and fission processes have also been shown to sensitize cells to apoptosis (17). The mitochondrial morphology in the U251 and U87 cells transitioned from mitochondrial fusion to fission after exposure to NC. Annexin V/PI staining was consistent with GBM cells undergoing apoptosis upon NC treatment. Recent research has shown that the release of cytochrome $c$ is initiated by the rupture of the outer mitochondrial membrane after an increase in Bax protein levels, which initiates apoptosis (24). Thus, the release of cytochrome $c$ and the increase in Bax protein are considered indicators of mitochondrial apoptosis. Cytochrome $c$ release and increases in Bax protein levels were observed in the NC-treated GBM cells. These results corroborate previous studies, that $\mathrm{NC}$ induces apoptosis in various cancer cells $(7,9,20,21,25,26)$. Apoptosis is thus an additional attribute that may contribute to the efficacy of NC in the treatment of human cancer.

Inhibition of tumor growth through these different cellular functions led us to examine the PI3K/Akt/mTOR signaling pathway as a potential target of NC. The PI3K/Akt/mTOR signaling cascades regulate a wide variety of cellular processes, such as cell proliferation, differentiation, survival, cell transformation and metastasis of tumor cells (27), and mutations in the PTEN tumor-suppressor gene, a critical regulator of $\mathrm{PI} 3 \mathrm{~K} / \mathrm{Akt} / \mathrm{mTOR}$, lead to unregulated activity of the pathway in diverse cancers. In our in vitro system, p-Akt and p-mTOR levels were decreased in the NC-treated GBM cells (Fig. 5A). p-Akt and p-mTOR levels were however partially restored in the presence of SC79, a novel Akt activator, as were functional activities including proliferation, invasion and apoptosis. Taken together, PI3K/Akt/mTOR signaling provides a molecular basis for the inhibition of tumor growth by $\mathrm{NC}$.

In summary, our data indicate that NC inhibits malignant behavior of GBM cells by targeting PI3K/Akt/mTOR signaling. NC thus warrants further investigation as a natural bioactive molecule with cancer-killing potential.

\section{Acknowledgements}

The present study was supported by the Natural Science Foundation of China grant (nos. 81402060 and 8157248), the Special Foundation for Taishan Scholars (nos. ts20110814 and tshw201502056), the Shandong Provincial Science and Technology Major Project (emerging industry) (2015ZDXX0801A01), the Fundamental Research Funds of Shandong University (2015QY001), the Key Research and Development Program of Shandong Province (nos. 2015GSF118074 and 2015GGE27101), the Department 
of Science and Technology of Shandong Province (nos. 2015GGE27101 and 2015ZDXX0801A01), the University of Bergen, the Helse Bergen, Norway and the Norwegian Centre for International Cooperation in Education (SIU) (UTF-2014/10047).

\section{References}

1. Ricard D, Idbaih A, Ducray F, Lahutte M, Hoang-Xuan K and Delattre JY: Primary brain tumours in adults. Lancet 379 : 1984-1996, 2012.

2. Stupp R, Brada M, van den Bent MJ, Tonn JC and Pentheroudakis G; ESMO Guidelines Working Group: High-grade glioma: ESMO Clinical Practice Guidelines for diagnosis, treatment and follow-up. Ann Oncol 25 (Suppl 3) iii93-iii101, iii2014

3. Van Meir EG, Hadjipanayis CG, Norden AD, Shu HK, Wen PY and Olson JJ: Exciting new advances in neuro-oncology: The avenue to a cure for malignant glioma. CA Cancer J Clin 60: 166-193, 2010.

4. Gordaliza M: Natural products as leads to anticancer drugs. Clin Transl Oncol 9: 767-776, 2007.

5. Newman DJ, Cragg GM and Snader KM: The influence of natural products upon drug discovery. Nat Prod Rep 17: 215-234, 2000.

6. Fang Z, Tang Y, Jiao W, Xing Z, Guo Z, Wang W, Shi B, Xu Z and Liu Z: Nitidine chloride inhibits renal cancer cell metastasis via suppressing AKT signaling pathway. Food Chem Toxicol 60: 246-251, 2013

7. Lin J, Shen A, Chen H, Liao J, Xu T, Liu L, Lin J and Peng J: Nitidine chloride inhibits hepatic cancer growth via modulation of multiple signaling pathways. BMC Cancer 14: 729, 2014.

8. Sun X, Lin L, Chen Y, Liu T, Liu R, Wang Z, Mou K, Xu J, Li B and Song H: Nitidine chloride inhibits ovarian cancer cell migration and invasion by suppressing MMP-2/9 production via the ERK signaling pathway. Mol Med Rep 13: 3161-3168, 2016.

9. Zhai H, Hu S, Liu T, Wang F, Wang X, Wu G, Zhang Y, Sui M, Liu $\mathrm{H}$ and Jiang L: Nitidine chloride inhibits proliferation and induces apoptosis in colorectal cancer cells by suppressing the ERK signaling pathway. Mol Med Rep 13: 2536-2542, 2016.

10. Javadov S, Baetz D, Rajapurohitam V,Zeidan A, Kirshenbaum LA and Karmazyn M: Antihypertrophic effect of $\mathrm{Na}^{+} / \mathrm{H}^{+}$exchanger isoform 1 inhibition is mediated by reduced mitogen-activated protein kinase activation secondary to improved mitochondrial integrity and decreased generation of mitochondrial-derived reactive oxygen species. J Pharmacol Exp Ther 317: 1036-1043, 2006.

11. Bettum IJ, Gorad SS, Barkovskaya A, Pettersen S, Moestue SA, Vasiliauskaite K, Tenstad E, Øyjord T, Risa Ø, Nygaard V, et al: Metabolic reprogramming supports the invasive phenotype in malignant melanoma. Cancer Lett 366: 71-83, 2015.

12. Keunen O, Johansson M, Oudin A, Sanzey M, Rahim SA, Fack F, Thorsen F, Taxt T, Bartos M, Jirik R, et al: Anti-VEGF treatment reduces blood supply and increases tumor cell invasion in glioblastoma. Proc Natl Acad Sci USA 108: 3749-3754, 2011.
13. Warburg O: On the origin of cancer cells. Science 123: 309-314, 1956.

14. Gao Y, Su Y, Qu L, Xu S, Meng L, Cai SQ and Shou C: Mitochondrial apoptosis contributes to the anti-cancer effect of Smilax glabra Roxb. Toxicol Lett 207: 112-120, 2011.

15. Youle RJ and van der Bliek AM: Mitochondrial fission, fusion, and stress. Science 337: 1062-1065, 2012.

16. Kasahara A and Scorrano L: Mitochondria: From cell death executioners to regulators of cell differentiation. Trends Cell Biol 24: 761-770, 2014.

17. Zhao J, Lendahl U and Nistér M: Regulation of mitochondrial dynamics: Convergences and divergences between yeast and vertebrates. Cell Mol Life Sci 70: 951-976, 2013.

18. Jo H, Mondal S, Tan D, Nagata E, Takizawa S, Sharma AK, Hou Q, Shanmugasundaram K, Prasad A, Tung JK, et al: Small molecule-induced cytosolic activation of protein kinase Akt rescues ischemia-elicited neuronal death. Proc Natl Acad Sci USA 109: 10581-10586, 2012

19. Liu N, Li P, Zang S, Liu Q, Ma D, Sun X and Ji C: Novel agent nitidine chloride induces erythroid differentiation and apoptosis in CML cells through c-Myc-miRNAs axis. PLoS One 10: e0116880, 2015.

20. Ou X, Lu Y, Liao L, Li D, Liu L, Liu H and Xu H: Nitidine chloride induces apoptosis in human hepatocellular carcinoma cells through a pathway involving p53, p21, Bax and Bcl-2. Oncol Rep 33: 1264-1274, 2015

21. Sun M, Zhang N, Wang X, Cai C, Cun J, Li Y, Lv S and Yang Q: Nitidine chloride induces apoptosis, cell cycle arrest, and synergistic cytotoxicity with doxorubicin in breast cancer cells. Tumour Biol 35: 10201-10212, 2014.

22. Pan X, Han H, Wang L, Yang L, Li R, Li Z, Liu J, Zhao Q, Qian M, Liu M, et al: Nitidine Chloride inhibits breast cancer cells migration and invasion by suppressing c-Src/FAK associated signaling pathway. Cancer Lett 313: 181-191, 2011.

23. Pérez-Escuredo J, Dadhich RK, Dhup S, Cacace A, Van Hée VF, De Saedeleer CJ, Sboarina M, Rodriguez F, Fontenille MJ, Brisson L, et al: Lactate promotes glutamine uptake and metabolism in oxidative cancer cells. Cell Cycle 15: 72-83, 2016.

24. Knudson CM and Brown NM: Mitochondria potential, bax 'activation,' and programmed cell death. Methods Mol Biol 414: 95-108, 2008.

25. Kang M, Ou H, Wang R, Liu W and Tang A: The effect of nitidine chloride on the proliferation and apoptosis of nasopharyngeal carcinoma cells. J BUON 19: 130-136, 2014.

26. Wang J, Wu J, Wu H, Liu X, Chen Y, Wu J, Hu C and Zou D: Liraglutide protects pancreatic $\beta$-cells against free fatty acids in vitro and affects glucolipid metabolism in apolipoprotein $\mathrm{E}^{-/}$ mice by activating autophagy. Mol Med Rep 12: 4210-4218, 2015.

27. Manning BD and Cantley LC: AKT/PKB signaling: Navigating downstream. Cell 129: 1261-1274, 2007. 